\title{
Pricing and Promotional Decisions of a Drug Manufacturer with the Presence of a Price-Volume Agreement
}

\author{
Hui Zhang ${ }^{1} \&$ Deanna Di Gregorio ${ }^{1}$ \\ ${ }^{1}$ The Faculty of Business Administration, Lakehead University, Thunder Bay, Ontario, Canada \\ Correspondence: Hui Zhang, The Faculty of Business Administration, Lakehead University, Thunder Bay, Ontario, \\ P7B 5E1, Canada. Tel: 1-807-343-8763.
}

Received: January 16, 2016

Accepted: February 3, 2016

Online Published: March 1, 2016

doi:10.5430/ijba.v7n2p1

URL: http://dx.doi.org/10.5430/ijba.v7n2p1

This paper is funded by The Natural Sciences and Engineering Research Council of Canada (NSERC).

\begin{abstract}
Physicians prescribe not only based on the health outcome and costs of a drug, but also based on their personal experience and preference for certain drugs. Thus, drug manufacturers invest a significant amount of promotional efforts to influence physicians, efforts including detailing, invited conferences, and sponsored research, etc. Since a third-party payer must pay for all claims for a drug that is covered by an insurance plan, the expense for the drug may be very high and uncertain. To control increasing drug costs and sales uncertainty, price-volume agreements have been proposed as a way to reduce the risk of higher-than-expected drug expenses. In such agreements, a drug manufacturer has to return a portion to the payer of sales exceeding a pre-specified volume threshold. In this paper, we investigated the drug pricing and promotional decisions of a drug manufacturer in the presence of a price-volume agreement under three different pricing scenarios. We found it not necessarily true that a high drug price or a high quality drug (with a high health benefit) reduces the manufacturer's motivation to promote. We also found that the existence of a price-volume agreement may not increase the drug price, but it does help control the promotional effort. Although a negotiated price may be lower than the price set by the manufacturer, a negotiation may not always be preferred because the manufacturer's profit is reduced by the negotiation and thus an agreement may not be reached under certain circumstances.
\end{abstract}

Keywords: promotions, detailing, price-volume agreement, pharmaceutical, cost-effectiveness

\section{Introduction}

The rising costs of healthcare in Canada and around the world continue to be a great concern to policy makers and the general population. In 2013, the total healthcare expenditure, as a percentage of Gross Domestic Product (GDP), was $10.2 \%$ in Canada and $16.4 \%$ in the United States (OECD, 2016). Of this healthcare spending, pharmaceuticals comprised the second largest component. Per capita spending on hospitals and physicians may have declined over time, while spending on pharmaceuticals continued to increase. In 2012, the Canadian Institute for Health Information (CIHI) estimated that $13.4 \%$ of Canadian healthcare spending was allocated to prescribed drugs (Canadian Institute for Health Information, 2014).

Risk-sharing agreements (RSAs) are one method used to control rising prescription drug expenses. A RSA is a common type of arrangement between a drug manufacturer and a third-party payer (i.e., private insurance company, public health plan, etc.). Price-volume agreements are one major form of a RSA, which targets markets with sales of drugs that are highly uncertain and thus the reimbursement by insurers may be high and uncertain (Rickard, 2002). A price-volume agreement is a variation of a volume-control method that links a drug's reimbursement price to a volume threshold. In this type of agreement, the manufacturer and payer agree on a specific volume threshold; if this threshold is reached (either in units or dollars, whichever is agreed to in the contract), the payer is reimbursed a proportion of revenues from the manufacturer for sales exceeding the established threshold (Willison \& Wiktorowicz, 2001). Various forms of price-volume agreements have been widely used in France, Italy, and Australia.

The uncertainty of the sales volume is exacerbated by both the manufacturer's promotional efforts and the physicians' prescribing behavior. Since the treatment decision is mainly made by physicians, drug manufacturers have invested a 
large amount in influencing physicians. Promotions directed at physicians can take various forms, such as sending sales representatives to visit physicians (detailing), inviting physicians to attend seminars and conferences, sponsoring physicians' research, etc. We use "detailing" to represent all promotional activities directed at physicians. Detailing_may impact physicians' preference for certain drugs. However, it may not be able to influence the payer's or the patients' preference.

There are multiple methods determining the reimbursement price of a drug listed on a formulary. Usually, the manufacturer proposes a price to the payer, and the payer decides to take it or negotiate over it. The price negotiation may take place separately from the price-volume negotiations. In some countries, prices are controlled by a regulatory authority other than the formulary such as the Patented Medicine Prices Review Board (PMPRB) in Canada. Thus, the price is an exogenous parameter. In some cases, the formulary price is based on the prices of similar drugs in other jurisdictions (reference pricing) and thus is not a term of the negotiation for the agreement. The payer sometimes may use the manufacturer's price for the reimbursement if the price is not considered excessive. It is common for the payer and the manufacturer to negotiate over the terms of the agreement for several rounds, and thus the price can be a bargaining factor.

Once a drug is included on a formulary, the payer has obligations to cover all claims under its limited use criteria. When a patient visits a physician for prescription drugs, the physician will provide treatment based on considerations such as the efficacy, safety, side effects of the drug, the patient's clinical status, and insurance policy -- as well as the patient's preference and the physician's own preference and experience with the treatment. The ultimate goal of the prescription is to help patients find the most cost-effective treatment.

Cost-effectiveness analysis has been used by physicians in making treatment decisions and by payers in formulary listing decisions. Thus, it can be viewed from the payer's perspective or from the physician's perspective. Although the physician's perceived health benefits of the drug can be influenced by the manufacturer's detailing effort, the payer's perception may not. Thus, the calculations of the cost-effectiveness for the payer and the physician may be different. In this paper, we consider both situations -- where the payer and physician's valuations of drug health benefit are and are not the same.

The purpose of this paper is to examine the impact of a price-volume agreement on the price and detailing decisions as well as on the manufacturer's profit and the cost-effectiveness of drug uses. We considered three different pricing scenarios: the price as an exogenous decision regulated by a government agency; the price as a decision set by the drug manufacturer; the price as a negotiated solution between the payer and the manufacturer. We tried to answer the following questions. What are the optimal drug price and the optimal detailing effort of the manufacturer? How do other parameters impact the manufacturer's detailing effort? What major differences exist between the different pricing scenarios for the optimal decisions and performance? What role does the price-volume agreement play in containing drug costs under different pricing scenarios?

\section{Literature Review}

Physician detailing is a marketing effort employed by manufacturers in order to educate physicians about the details of a particular drug, including the scientific information, benefits, and side effects. Along with drug information, some manufacturers also provide physicians with gifts and free drug samples. Another type of detailing that is increasing in popularity as technology advances is e-detailing. This involves the use of social networking and other online technologies by pharmaceutical representatives and physicians to build a relationship and exchange pharmaceutical information.

Current literature contains a large amount of documentation about detailing and the impact that such efforts have on physicians. As the existing literature suggests, detailing efforts can have a profound influence on a physician's decision to prescribe and his or her preference for a particular drug. Mizik (Mizik \& Jacobson, 2004) found that physician detailing and complimentary drug samples both have a positive and statistically significant effect on the prescribing behaviors of physicians. These results are strongly grounded in past research, as several studies found similar findings (Bingle, O'Connor, Evans, \& Detamore, 1991, Wazana, 2000, Paris \& Docteur, 2006).

In addition to the positive influence on a physician's frequency of prescribing a drug, detailing has also been found to have a positive and significant impact on a physician's choice of drug to prescribe. Donohue and Berndt (Donohue \& Berndt, 2004) found that drug choice probability positively increases with an increase in detailing expenses. Physician detailing has a significant and lasting influence on a physician's drug choice (Iizuka \& Jin, 2007, Søndergaard, Vach, Kragstrup, \& Andersen, 2009, Grundy, Bero, \& Malone, 2013). Manchanda and Chintagunta 
(2004) reported that physicians who were over-detailed prescribed $50 \%$ more than the average physician, while physicians who were optimally detailed prescribed $60-104 \%$ more.

The impact of detailing on physicians' prescribing behavior -- as Montoya, Netzer, and Jedidi (2010) have discovered -- may not only occur in the short-term but also in a long-term. Montoya et al. (2010) found that detailing had only a small impact on a physician in the short-term but had a much stronger, long-lasting effect on the physician's writing of new prescriptions. When detailing was coupled with the distribution of samples, an even greater retention strategy was employed.

Several theoretical models have been developed on the optimal decision making under a price-volume agreement (Zhang \& Zaric, 2011, Zhang, Zaric, \& Huang, 2011, Zhang \& Zaric, 2015). Zhang et al. (2011) investigated how a price-volume agreement can be optimally designed with the presence of uncertain demand. They assumed that the drug manufacturer has private information about the expected demand and reports it to the payer in the negotiation of the agreement. The payer thus designs a menu of contracts including the drug price and the rebate rate as functions of the reported demand to induce the manufacturer to reveal its true value. In this model, they did not include the promotional effort by the manufacturer and considered only the case where the payer chooses the drug price. Zhang and Zaric (2011) and Zhang and Zaric (2015) included the promotional decision of the drug manufacturer, but their studies are different from ours in several aspects. First, the impact of the promotional effort on the demand took a different form. They assumed that promotions increase sales in a linear form while we assumed that promotions change physicians' preference and thus the prescriptions. Second, they considered different pricing scenarios than ours, and none of them considered the price as a Nash bargaining solution. Third, this paper investigated the impact of different pricing scenarios on both pricing and promotional decisions and identified whether a price scenario is superior to the others.

Besides these studies on price-volume agreements, there are several theoretical studies on the risk-sharing contracts in controlling uncertainties in pharmaceutical market (Zaric \& Xie, 2009, Antonanzas, Juarez-Castello, \& Rodriguez-Ibeas, 2011, Barros, 2011), which, however, are mainly focused on the uncertainty of drugs' health outcomes on patients. In particular, Zaric and Xie (2009) compared two forms of risk-sharing agreements under a two-period setting; Antonanzas et al. (2011) assumed that the drug price is negotiated between a drug manufacturer and the payer, and Barros (2011) assumed that the manufacturer sets a drug price.

\section{Model Structure and Assumptions}

We considered a setting in which a physician prescribes a brand-name drug to a patient based on the drug's cost-effectiveness analysis and his preference (Wright, 2004). The physician may have other alternatives to choose in the same therapeutic class, or if not, the physician may choose not to provide any treatment if the drug does not match the patient's diagnosis status. The physician's valuation of the drug's health benefit may be influenced by the drug manufacturer's detailing activities. Although the physician may get some information and suggestions from the patient about the treatment, it is the physician's ultimate decision as to whether or not to prescribe the drug. Let $b$ represent the original incremental net health benefits excluding drug cost in monetary terms that a patient may receive by consuming one unit of a drug. Let $m$ denote the additional health benefits brought about by detailing, i.e., the detailing effect on drug outcomes. In other words, $m$ is the increase in health benefits that a physician perceives as a result of receiving promotional information from the drug manufacturer. Detailing increases the perceived health benefits because a physician becomes more aware of the drug's benefits and risks and thus can prescribe the drug more properly; or because a physician is impacted by the detailing effort and thus has an increased preference for the drug. In the remaining part of the paper, we used $m$ to represent the level of detailing effort. The perceived health benefit with detailing is thus $m+b$. This form of increased health benefit was also used by Barros (2011). We assumed that the detailing cost takes the form of $\mathrm{am}^{2} / 2$, a convex function reflecting the diminishing effect between detailing efforts and prescribing behavior (Gonul, Carter, Petrova, \& Srinivasan, 2001). In this form, $a>0$ is a parameter representing the size of detailing effort by increasing a certain level of the physician's perception of the health benefit.

The physician may have a preference for the drug, denoted by $\theta$. $\theta$ is a random variable drawn from a uniform distribution in $\left[\theta_{0}, \theta_{0}+1\right]$, where $\theta_{0} \geq 0$ represents the level of preference by the physician and the degree of heterogeneity among physicians. When $\theta_{0}<1$ different physicians are significantly heterogeneous with respect to their preference. Thus, the perceived health benefit with the physician's preference is $\theta(m+b)$, with a similar form used by Wright (2004).

Let $p$ represent the unit price of the drug being sold to the payers. Thus, the physician's perception of the cost-effectiveness of the drug is $\theta(m+b)-p$. The physician makes prescribing decisions by comparing the 
cost-effectiveness of the drug with other alternatives. To simplify the situation, we normalized that the cost-effectiveness of the other alternatives (including no treatment) as zero. That is, he prescribes only when the net health benefit (NMB) of the drug is non-negative, (i.e., when $\theta(m+b)-p \geq 0$ or $\theta \geq p /(m+b))$. NMB is the total health benefit of drug uses in monetary terms minus the total cost of the drug and is used to measure whether a treatment is cost-effective.

Let $Q$ be the total potential demand or requests (in drug units) from the patients, and only a portion of the demand is satisfied by the doctor due to the concern of cost-effectiveness. The prescribed amount is thus $Q\left(\theta_{0}+1-p /(m+b)\right)$, where $1 /(m+b)$ represents the price elasticity of the demand. The total demand of the drug is $N=Q\left(\theta_{0}+1-p /(m+b)\right)+\varepsilon$, where $\varepsilon$, a random variable, is drawn from a normal distribution with a mean of 0 and standard deviation of $\sigma$.

Let $T$ represent the volume threshold in a price-volume agreement, which is assumed to be exogenously determined. In a price-volume agreement, the manufacturer and the payer may agree upon a threshold based on the manufacturer's estimate of sales (Paris \& Docteur, 2006). If the total sales exceed the threshold value, the manufacturer pays a proportion, $\alpha, 0 \leq \alpha \leq 1$, of the excess sales to the payer. The rebate rate $\alpha$ is assumed to be exogenous and in some instances it is set as $100 \%$ (Adamski et al., 2010). Therefore, the manufacturer is paid the unit price $p$ until the threshold $T$ is reached; the payment is reduced to $(1-\alpha) p$ for any sales above $T$. This reduction is in the form of a rebate, where the manufacturer is initially paid in full $p$ but then must return a proportion, $\alpha$, of exceeding sales to the payer.

Let $E[\pi]$ denote the manufacturer's expected profit, which includes the total revenue generated from the sales and excludes the rebate to the payer as well as any marketing costs. Although research and development costs are also incurred, these are sunk costs and can be ignored. Therefore, the manufacturer's profit is $E[\pi]=(p-k) E[N]-\alpha p E\left[(N-T)^{+}\right]-a m^{2} / 2$, where $k, 0<k<p$ is the marginal manufacturing cost of the drug, and $(N-T)^{+}=\max (0, N-T)$.

\section{Analysis and Results}

We investigated how the detailing and pricing decisions are made under three different pricing scenarios.

\subsection{Case (E) The Price Is Exogenously Determined}

When the price is exogenously determined, the manufacturer chooses the detailing effort to maximize its expected profit. Given a price $p$, the manufacturer's problem in case (E) is stated as follows:

$$
\begin{aligned}
& \max _{m} E[\pi] \\
& \text { s.t. } \quad m \geq 0 .
\end{aligned}
$$

The first-order and second-order conditions of the manufacturer's profit with respect to the detailing effort are

$$
\frac{d E[\pi]}{d m}=\frac{Q p^{2}}{(m+b)^{2}}\left[1-\alpha(1-F)-\frac{k}{p}\right]-a m,
$$

and

$$
\frac{d^{2} \mathrm{E}[\pi]}{d m^{2}}=-\frac{2 Q p^{2}}{(m+b)^{3}}\left[1-\alpha(1-F)-\frac{k}{p}\right]-\frac{Q \alpha p^{3}}{(m+b)^{4}} f-a<0,
$$

where $F \equiv F\left(T+Q\left(\frac{p}{m+b}-\theta_{0}-1\right)\right)$ and $f \equiv f\left(T+Q\left(\frac{p}{m+b}-\theta_{0}-1\right)\right)$.

Since the manufacturing cost of pharmaceutical drugs is usually low, we assume that $k$ is sufficiently small to guarantee $1-\alpha(1-F)-k / p \geq 0$. Otherwise, the expected profit is decreasing with $m$, and the optimal $m$ is 0 . 
Under this assumption, there is a unique optimal solution, denoted by $m^{E, *}$, to the drug manufacturer. The optimal detailing effort is obtained from

$$
\frac{Q p^{2}}{(m+b)^{2}}\left[1-\alpha(1-F)-\frac{k}{p}\right]-a m=0 .
$$

The properties of the optimal detailing effort are summarized in the following proposition.

\section{Proposition 1.}

(1). $-1<\frac{\partial m^{E, *}}{\partial b}<0, \frac{\partial m^{E, *}}{\partial p}>0, \frac{\partial m^{E, *}}{\partial a}<0, \frac{\partial m^{E, *^{*}}}{\partial \theta_{0}}<0, \frac{\partial m^{E, *}}{\partial k}<0, \frac{\partial m^{E, *}}{\partial \alpha}<0$ and $\frac{\partial m^{E, *}}{\partial T}>0$.

(2). $\frac{\partial E\left[\pi^{E, *}\right]}{\partial b}>0, \frac{\partial E\left[\pi^{E, *}\right]}{\partial p}$ may be positive or negative, $\frac{\partial E\left[\pi^{E, *}\right]}{\partial a}<0, \frac{\partial E\left[\pi^{E, *}\right]}{\partial \theta_{0}}>0, \frac{\partial E\left[\pi^{E, *}\right]}{\partial k}<0$, $\frac{\partial E\left[\pi^{E, *}\right]}{\partial \alpha}<0$ and $\frac{\partial E\left[\pi^{E, *}\right]}{\partial T}>0$.

When facing a higher price, the drug manufacturer tends to promote more intensively. It is counterintuitive because price is usually perceived as a signal of quality. When only cost-effective drugs are prescribed, it is expected that the higher priced drugs have higher quality and thus the drug manufacturer does not necessarily put too much effort into promoting high quality products. The counterintuitive result is because the price is exogenously determined in this case. It may be true that, when price is a decision variable, the manufacturer will set a higher price for better quality products and thus the drug price is a symbol of drug quality. In this case, the manufacturer tries to increase promotions for more expensive drugs to gain more profit because the marginal benefit of increasing sales rises with the price. Thus, we have to note that expensive drugs neither signal high quality drugs nor generate high profit for the manufacturer in a setting with regulated prices. In the next section we explored the situation in which price can be a decision variable to investigate whether higher quality leads to higher price.

\subsection{Case (M) The Price Is Also a Decision by the Manufacturer}

In some cases, the manufacturer sets the drug price and the payer may simply use the proposed price as the reimbursement price. A similar example that assumes the price is the manufacturer's decision is found in Barros (2011). Although we assumed that each prescription is based on cost-effectiveness analysis, the overall use of a drug may not be cost-effective, that is, the expected NMB may be negative. The expected NMB is $E[N M B]=b E[N]-\left\{p E[N]-\alpha p E\left[(N-T)^{+}\right]\right\}$, where the first part is the total health benefit in monetary terms, and the second part is the transfer cost from the payer to the manufacturer. Another approach to measure the NMB may use the marketing-induced health benefit $(m+b)$ instead of using the original health benefit $b$. In this form, the NMB is from the manufacturer's or the physician's perspective, rather than from the payer's perspective.

The manufacturer sets its price and reports its future detailing efforts before entering the price-volume agreement. Once the agreement is reached between the manufacturer and the payer, the manufacturer then chooses its detailing effort to maximize its expected profit. The problem can be solved in a backward induction. That is, the manufacturer first chooses its promotional effort $m$, which is obtained from equation (1); then it set its drug price by anticipating its future effort. The manufacturer's problem in (M) is thus stated as follows.

$$
\max _{p} E[\pi]
$$

s.t. $\quad(1), p \geq 0$ 
Since the physician prescribes based on cost-effectiveness analysis, we thus did not include the NMB as a constraint in this formulation. The optimal marketing effect obtained from equation (1) is a function of the price $p$, denoted by $m(p)$, which is substituted into the manufacturer's profit function and the NMB constraint, and both functions become functions of the price $p$.

The first-order and second-order conditions of the profit as a function of $p$ are as follows.

$$
\frac{d E[\pi]}{d p}=E[N]-\alpha E\left[(N-T)^{+}\right]-\frac{Q p}{m+b}\left[1-\alpha(1-F)-\frac{k}{p}\right],
$$

and

$$
\begin{gathered}
\frac{d^{2} \mathrm{E}[\pi]}{d p^{2}}=-\frac{2 Q}{m+b}[1-\alpha(1-F)]-\frac{Q \alpha p f}{(m+b)^{2}}+\left\{\frac{2 Q p}{(m+b)^{2}}[1-\alpha(1-F)]-\frac{Q k}{(m+b)^{2}}+\frac{Q \alpha p^{2} f}{(m+b)^{3}}\right\} \frac{d m(p)}{d p} \\
=\frac{Q k^{2}}{(m+b)^{4}}-Q a\left\{\frac{2}{m+b}[1-\alpha(1-F)]+\frac{\alpha p f}{(m+b)^{2}}\right\} .
\end{gathered}
$$

\section{A Special Case When $k=0$}

Since it is difficult to prove the concavity of $E[\pi]$ with respect to $p$, we first considered a special case in which the marginal manufacturing cost $k$ is so low that it can be ignored in the manufacturer's profit function. It is true for many drugs in the pharmaceutical industry since compared to research and development, the production cost of the drug is relatively low and can be ignored in many cases. For that case, $d^{2} \mathrm{E}[\pi] / d p^{2}<0$, and it is obvious that there is a unique optimal price for the drug manufacturer, and the optimal price is obtained from the following equation:

$$
E[N]-\alpha E\left[(N-T)^{+}\right]-\frac{Q p}{m+b}[1-\alpha(1-F)]=0 .
$$

Thus, the optimal detailing effort and the optimal price are solutions to equations (1) and (2). We use $m^{M \text {,* }^{*}}$ and

$p^{M, *}$ to represent the optimal detailing effort and the optimal price of the manufacturer.

Proposition 2. When $k=0$, the optimal solutions of $m$ and $p$ and the resulting performance have the following properties.

(1). $\frac{d m^{M, *}}{d b}=0, \frac{d m^{M, *}}{d \theta_{0}}>0, \frac{d m^{M, *}}{d a}<0, \frac{d m^{M, *}}{d \alpha}<0$ and $\frac{d m^{M, *}}{d T}>0$.

(2). $\frac{d p^{M, *}}{d b}>0, \frac{d p^{M, *}}{d \theta_{0}}>0, \frac{d p^{M, *}}{d a}<0$, and $\frac{d p^{M, *}}{d \alpha}$ and $\frac{d p^{M, *}}{d T}$ may be positive or negative.

(3). $\frac{d E\left[\pi^{M, *}\right]}{d b}>0, \frac{d E\left[\pi^{M, *}\right]}{d \theta_{0}}>0, \frac{d E\left[\pi^{M, *}\right]}{d a}<0, \frac{d E\left[\pi^{M, *}\right]}{d \alpha}<0$ and $\frac{d E\left[\pi^{M, *}\right]}{d T}>0$.

Part (1) shows that the optimal detailing effort does not change as the health benefit changes. That is, the drug quality does not impact the promotional activity of the manufacturer. This is different from the observation obtained in proposition 1 in which the manufacturer has less motivation to promote high quality products when the price is exogenous. On the one hand, when the price is determined by the manufacturer, the manufacturer charges a higher price for a higher quality product and exerts more effort to promote a more expensive product. On the other hand, the manufacturer tends to reduce its effort in promoting a higher quality product. The combination of these two opposite 
effects results in the insensitivity of the optimal detailing effort with respect to the change in health benefits when the price is also a decision by the manufacturer.

When the manufacturer is confronted with a higher threshold, it has the incentive to promote more to earn a higher profit. However, the price may be reduced or increased, depending on many other factors. In the numerical experiments, we explored how these factors influence the manufacturer's pricing decision. The existence of a price-volume agreement may reduce the manufacturer's detailing effort and may not boost the drug price, which is different from the finding that a risk-sharing contract (with uncertain drug benefit) increases the drug price (Capri \& Levaggi, 2010).

\section{Numerical Examples}

In this section, we developed numerical experiments to illustrate how different parameters influence the optimal detailing effort and the optimal price for case (M). In the base case, we set $\alpha=1, \theta_{0}=0, b=30, T=50, a=1, k=1$, and $\varepsilon \sim N\left(0,10^{2}\right)$.

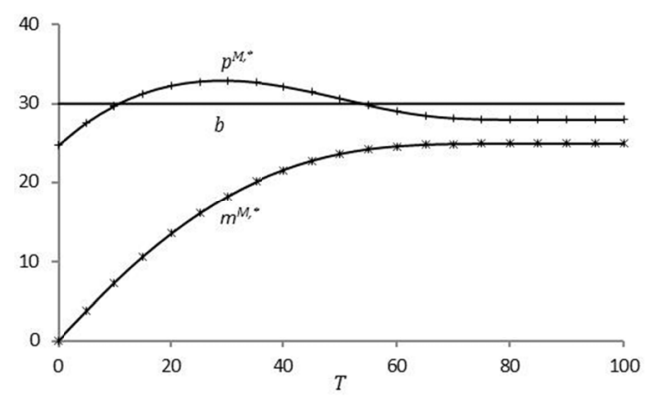

Figure 1a. The optimal detailing effort and the optimal price with the change of $T$

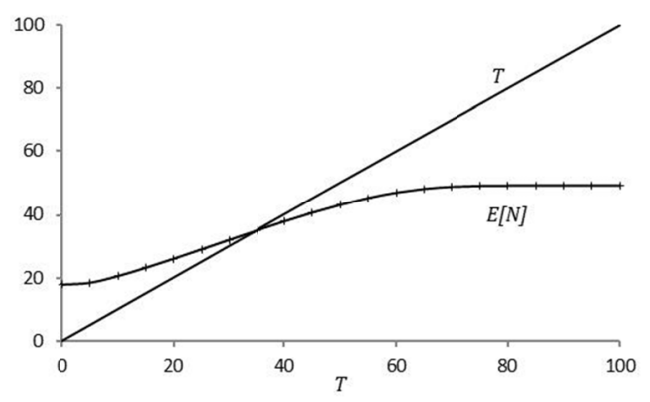

Figure $1 \mathrm{~b}$. The comparison between the expected demand $\mathrm{E}[N]$ and the threshold value $T$

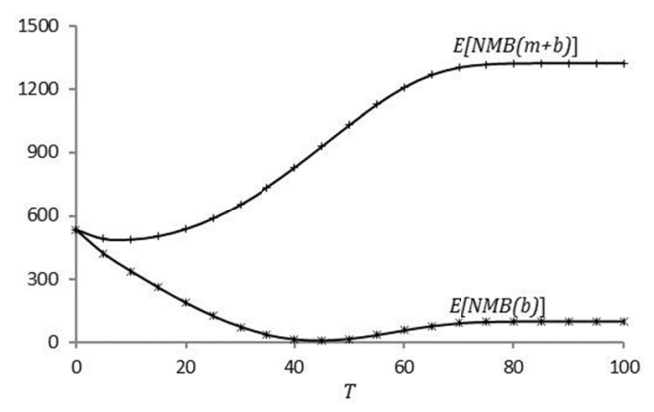

Figure 1c. $\mathrm{E}[\mathrm{NMB}]$ with respect to the change of $T$, where $\mathrm{E}[\mathrm{NMB}(\mathrm{b})]$ represents $\mathrm{E}[\mathrm{NMB}]$ when using the original health benefit $b$, and $\mathrm{E}[\mathrm{NMB}(m+b)]$ represents $\mathrm{E}[\mathrm{NMB}]$ when using the updated health benefit $(m+b)$ 
We first fixed other parameter values and changed the value of $T$. Figure 1a illustrates how the optimal detailing effort and the optimal price change with $T$. It is obvious that the optimal detailing effort increases with the threshold, as demonstrated in proposition 2. As the threshold rises to a high level, the optimal detailing effort will stabilize. This suggests that setting a high threshold value does not necessarily induce excessive detailing efforts and that the payer should set the threshold value at a relatively low level if it tends to control the detailing effort by changing a threshold. The optimal price increases with the threshold and then declines and stays at relatively high threshold levels. When the threshold is relatively low $(T \leq 30)$, the optimal detailing effort quickly increases with $T$ and, accordingly, the price elasticity of demand decreases. The reduced sensitivity of demand to price motivates the manufacturer to set a higher price. That is, when $T$ increases at relatively low level, the manufacturer has incentives to set a higher price and promote more aggressively. As the threshold continues increasing after a certain point, the demand becomes less sensitive to price change, and the price is expected to get higher. However, this is not true in this example. This occurs likely because, as the threshold rises to a high level, the expected demand slows down and is lower than the rising threshold value (Figure 1b) so that the expected rebate becomes larger and the manufacturer has to set a lower price to compensate the increasing return. One implication to the payer is that setting a high threshold value may not help control the manufacturer's detailing effort, but it does help control the drug price.

Figure 1a also shows that the optimal price may be higher or lower than the original health benefit $b$. That is, without the physician's preference and impact of detailing efforts, the prescription is cost-effective for a relatively low or a relatively high threshold, while not being cost-effective otherwise. This results in a decreasing E[NMB] with $T$ when $T$ is relatively low and an increasing E[NMB] with $T$ when $T$ is relatively large (T>45) (shown in Figure 1c). Figure 1c suggests that the overall drug uses may be most cost-effective at a very low threshold value. This is because a low threshold helps prevent detailing efforts, which may impact the physician's valuation of the drug benefit but does not necessarily change the payer's valuation. Thus, with the presence of heavy detailing efforts, requiring cost-effective prescriptions may not guarantee that the overall treatment is cost-effective. If the payer's valuation is also impacted by the detailing effort, then E[NMB] benefits from the increasing threshold, and the overall prescriptions are cost-effective.

We changed the value of $k$ within the range of $[0, b)$. It is straightforward that the optimal price increases with the manufacturing cost. However, this increase may not be as fast as the change of the manufacturing cost. Figure $2 \mathrm{a}$ shows that the optimal detailing effort decreases with the marginal manufacturing cost in order to control the total manufacturing expenses. That is, the manufacturer will set a higher price but control its detailing effort in cases of higher manufacturing cost. The rising manufacturing cost does not only decrease the expected profit but also may reduce the expected NMB for the payer (Figure 2b). If the payer's valuation of the health benefit is not changed by the detailing effort, the expected NMB may be negative because $b<p$. E[NMB], with the original health benefit, first decreases but finally increases with $k$ because, as the price increases with the manufacturing cost, the rising rebate finally contributes to the increase of NMB at a relatively high $k$. If the payer's valuation of the health benefit is also impacted by the detailing effort, then the expected NMB decreases with the rising manufacturing cost because of decreasing detailing effort.

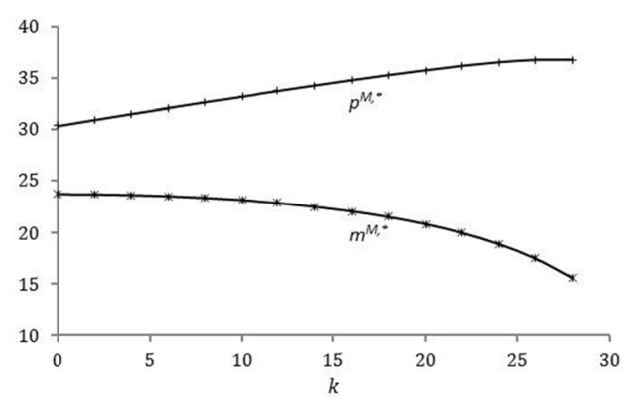

Figure 2a. The optimal price and detailing effort with respect to the change of $k$ 


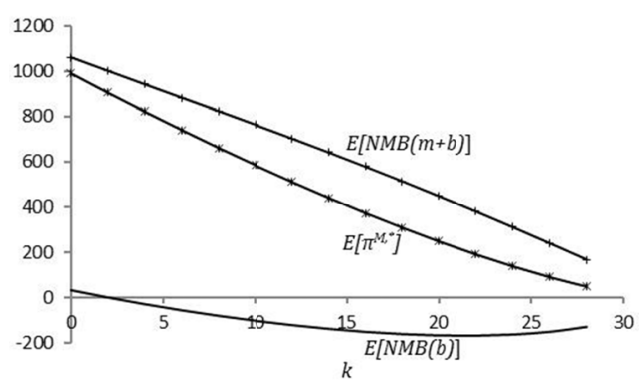

Figure $2 \mathrm{~b}$. The expected profit and NMB with respect to the change of $k$

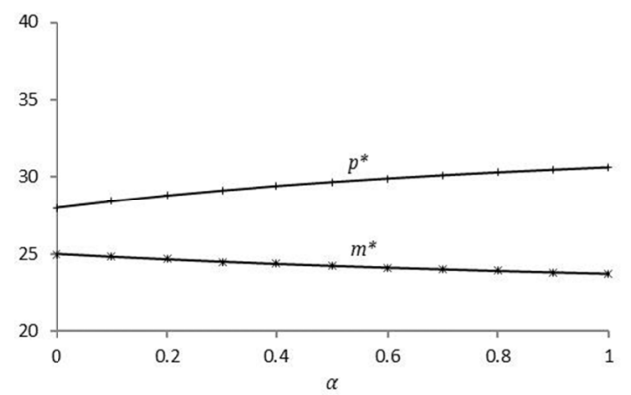

Figure 3a. The optimal price and detailing effort with respect to the change of $\alpha$

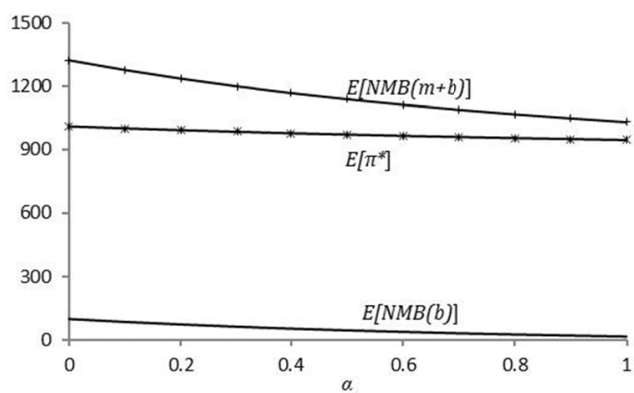

Figure $3 b$. The optimal profit and expected NMB with respect to the change of $\alpha$

With the increasing penalty on excess sales beyond the threshold value, the manufacturer would set a higher price and cut its input on detailing, as shown in Figure 3a. It is expected that the manufacturer's profit will decline with the rising rebate rate. However, it is counterintuitive that the payer's NMB will also decline (Figure 3b). A higher price discourages the physician from prescribing and thus generates less demand. As the price rises, the net health benefits decrease so that an increased return may not be large enough to guarantee a rising NMB with respect to the rebate rate. Imposing a price-volume agreement may help with controlling the promotional activities, but it may not result in more cost-effective drug uses or an improvement in the total welfare, represented by the sum of the manufacturer's profit and the payer's NMB.

\subsection{Case (N) When the Price Is Negotiated between the Payer and the Manufacturer}

When the drug price is negotiated between the payer and the drug manufacturer, the two parties reach an agreement on price based on their negotiation power, as in Antonanzas (2011). The negotiated price is thus a Nash bargaining solution. Given $m(p)$ from equation (1), the negotiated price is obtained from the following equation:

$$
\begin{array}{ll}
p^{N, *}=\underset{p}{\arg \max _{p}}\left\{(E[\pi])^{\beta}(E[N M B])^{1-\beta}\right\} . \\
\text { s.t. } \quad(1), m, p \geq 0, E[\pi] \geq 0, \mathrm{E}[\mathrm{NMB}] \geq 0 .
\end{array}
$$


$\beta$ is used to represent the negotiation power of the drug manufacturer. To ensure the existence of a negotiated price, the manufacturer has to obtain positive profit. We assumed that the payer's valuation of the drug benefit is not impacted by the detailing effort and thus use $\mathrm{E}[\mathrm{NMB}]$ with the original health benefit $b$. It is difficult to obtain the analytical solution to this problem. We thus referred to numerical examples to illustrate how the optimal decisions and resulting performance are influenced by other parameter values and how they are different from the optimal decisions and performance when the price is determined by the manufacturer. In the example, we set $\beta=0.5$, implying that both the manufacturer and the payer have the same negotiation power. We kept the other parameter values the same as in the base case.

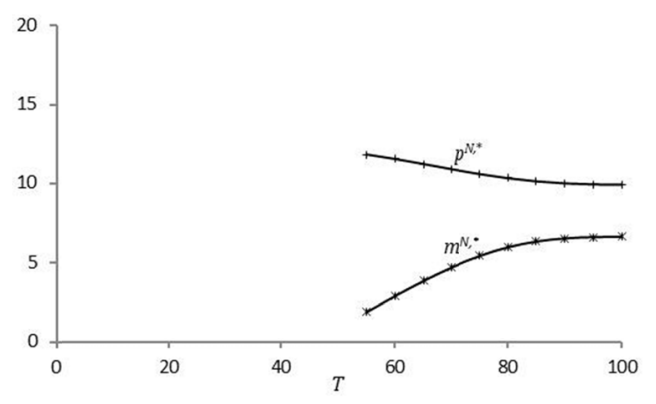

Figure 4. The optimal price and detailing effort change with various values of $T$

Figure 4 shows that, by increasing the threshold value, the payer would cut the negotiated price but has to allow more detailing effort. The change of the threshold value has a similar impact on the optimal price and detailing effort as in case (M), where the price is set by the manufacturer. It is not surprising to see that the negotiated price is much lower than the price set by the manufacturer. However, in a negotiation setting, it might be difficult to reach an agreement because the manufacturer cannot obtain a positive profit at relatively low threshold values and thus may be unwilling to sign the agreement. Once a negotiated price is reached, the manufacturer's profit is dramatically reduced while the payer's NMB is improved. The total welfare is increased by choosing the negotiation process. However, the negotiation may take several rounds, and is therefore costly and time-consuming to reach an agreement. In situations when innovative treatments are encouraged, it may not be a good idea to use a negotiated price for the regulator.

Figure 5 shows that the optimal price and detailing effort exhibit similar patterns to the change of $\alpha$ as when the price is set by the manufacturer, although the price and detailing effort are lower in the negotiation setting. A big difference is that the negotiation cannot reach an agreement when the rebate rate is high $(\alpha>0.8)$, mainly because the manufacturer's profit declines to negative values with a high penalty.

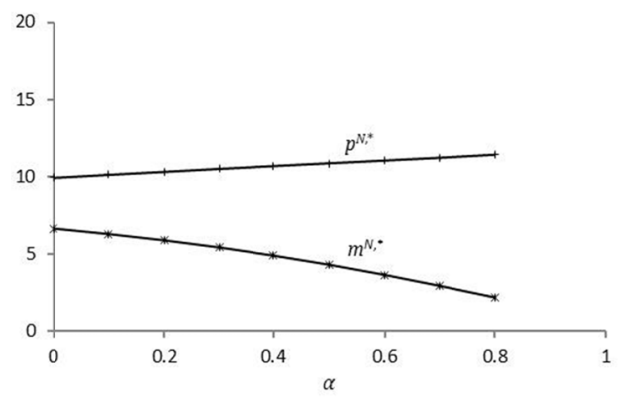

Figure 5. The optimal price and detailing effort with respect to the change of $\alpha$

\section{Discussion and Future Directions}

We have examined the impact of a price-volume agreement on drug manufacturers' detailing and pricing decisions in the pharmaceutical market under various pricing scenarios. Although several papers have discussed physician detailing and price-volume agreements or risk-sharing contracts, we have made unique contributions to the literature of theoretical models in drug pricing and marketing. First, we modelled both the pricing and promotional decisions with the presence of a price-volume agreement in a prescription drug market, while Barros (2011) briefly described a 
model with both pricing and detailing decisions under a risk-sharing contract with uncertain drug effectiveness. Second, we considered three different pricing scenarios in this paper, and we are unaware of any other studies which investigated all these pricing methods. Third, we combined the cost-effectiveness concerns of both the health provider and the payer while other studies may have considered only one of them.

Our analysis yields a number of interesting insights. First, we found that the drug price may not necessarily be the signal of drug quality, especially in the setting where the price is exogenously determined. Thus, an expensive drug may incent the manufacturer to promote more aggressively, rather than reducing promotion on more expensive drugs, which are perceived as high quality products in common sense. Second, imposing a price-volume agreement may not increase the drug price, although in our numerical experiments we did find higher prices with rising rebate rates. Third, although a price-volume agreement helps control detailing efforts, it does not necessarily encourage more cost-effective uses of the drug and not necessarily contribute to the social welfare when the price is set by the manufacturer. Fourth, a negotiated price helps with controlling the drug price as well as promotions and contributes to a higher social welfare. However, the payer has to exercise caution in the negotiation process for several reasons. It may be costly and time-consuming; it may be not able to reach an agreement as easily as in the other pricing scenarios; it reallocates the social welfare between the two parties and may sacrifice the manufacturer's profit as well as its incentives for innovation.

In extending this analysis to better understand its impact on the broader healthcare picture, it is essential to appreciate the importance of $\alpha$ on the results of this study. Imposing a price-volume agreement may incent a higher price, regardless of whether this price is set by the manufacturer or by negotiation. This observation is similar to the common concern that a risk-sharing contract will increase the drug price (Capri \& Levaggi, 2010). A price-volume agreement may help control the manufacturer's excess profit but may not be able to boost the payer's NMB and the total welfare. The payer has to exercise caution in applying the agreement, especially in the situation where physicians are price-sensitive and thus withhold their prescriptions of expensive drugs due to cost-effectiveness concerns.

Our paper has several limitations. We considered the manufacturer's promotional decisions in relation to physicians but did not include other marketing activities such as direct-to-consumer advertising and promotional efforts directed toward the payer. We assumed that the average health benefit is a constant while in practice it may be uncertain. We assumed that the physician will follow prescription principles and prescribe based on cost-effectiveness analysis but, in reality, some physicians may be concerned more with efficacy or cost. We assumed that detailing increases physicians' valuation of the drug while in practice too much detailing effort may negatively influence physicians' valuation of the drug benefit (Gonul et al., 2001).

We can extend this paper in various directions in the future. We considered only one drug from one manufacturer while normalizing the other options' NMB as zero. We may extend this to two drugs by two manufacturers when the more cost-effective drug is prescribed. We would assume both manufacturers promote to impact the physicians' preference for their own drugs. We may also extend this paper to include patients' influence on the prescription decision, in which the physician proposes a treatment and the patient may accept or decline.

\section{References}

Adamski, J., Codman, B., Ofierska-Sujkowska, G., Herholz, H., Wendykowska, K., Laius, O., ... Gustafsson, L. (2010). Risk sharing arrangements for pharmaceuticals: potential considerations and recommendations for European payers. BMC Health Service Research, 10(153), 1-26. http://dx.doi.org/10.1186/1472-6963-10-153

Antonanzas, F., Juarez-Castello, C. A., \& Rodriguez-Ibeas, R. (2011). Should health authorities offer risk-sharing contracts to pharmaceutical firms? A theoretical approach. Health Economics, Policy and Law, 6(3), 391-403. http://dx.doi.org/10.1017/S1744133111000016

Barros, P. P. (2011). The simple economics of risk-sharing agreements betwen the NHS and the pharmaceutical industry. Health Economics, 20(4), 461-470. http://dx.doi.org/10.1002/hec.1603

Bingle, G., O'Connor, T., Evans, W., \& Detamore, S. (1991). The effect of 'detailing' on physicians' prescribing behavior for postsurgical narcotic analgesia. Pain, 45(2), 171-173. http://dx.doi.org/10.1016/0304-3959(91)90185-Z

Canadian Institute for Health Information. (2014). Drug Spending in 2014. Retrieved October 08, 2015, from https://www.cihi.ca/web/resource/en/nhex_2014_infosheet_en.pdf 
Capri, S., \& Levaggi, R. (2010). Shifting the risk in pricing and reimbursement schemes. A new model of risk-sharing agreements for innovative drugs. Liuc Papers n. 234, Serie Economia e Impresa 63.

Donohue, J., \& Berndt, E. (2004). Effects of direct-to-consumer advertising on medication choice: the case of antidepressants. Journal of Public Policy \& Marketing, 23(2), 115-127. http://dx.doi.org/10.1509/jppm.23.2.115.51395

Gonul, F., Carter, F., Petrova, E., \& Srinivasan, K. (2001). Promotion of prescription drugs and its impact on physicians' choice behavior. Journal of Marketing, 65(3), 79-90. http://dx.doi.org/10.1509/jmkg.65.3.79.18329

Grundy, Q., Bero, L., \& Malone, R. (2013). Interactions between Non-Physician Clinicians and Industry: A Systematic Review. PLOS Medicine, 10(11), 1-13. http://dx.doi.org/10.1371/journal.pmed.1001561

Iizuka, T., \& Jin, G. Z. (2007). Direct-to-consumer advertising and prescription choice. The Journal of Industrial Economics, 51(2), 243-270. http://dx.doi.org/10.1111/j.1467-6451.2007.00329.x

Manchanda, P., \& Chintagunta, P. K. (2004). Responsiveness of physician prescription behavior to salesforce effort: an individual level analysis. Marketing Letters, 15(2-3), 129-145.

Mizik, N., \& Jacobson, R. (2004). Are physicians "easy marks"? Quantifying the effects of detailing and sampling on new prescriptions. Management Science, 50(12), 1704-1715. http://dx.doi.org/10.1287/mnsc.1040.0281

Montoya, R., Netzer, O., \& Jedidi, K. (2010). Dynamic allocation of pharmaceutical detailing and sampling for long-term profitability. Marketing Science, 29(5), 909-924. http://dx.doi.org/10.1287/mksc. 1100.0570

OECD. (2016). Health expenditure and financing. Retrieved January 17, 2016, from http://stats.oecd.org/Index.aspx?DataSetCode=SHA

Paris, V., \& Docteur, E. (2006). Pharmaceutical Pricing and Reimbursement Policies in Canada. OECD Working Papers.

Rickard, M. (2002). The pharmaceutical benefit scheme: Options for cost control. Parliament of Australia. Current Issues Briefs 2001 - 2002.

Søndergaard, J., Vach, K., Kragstrup, J., \& Andersen, M. (2009). Impact of pharmaceutical representative visits on GPs' drug preferences. Fam Pract, 26(3), 204-209. http://dx.doi.org/10.1093/fampra/cmp010

Wazana, A. (2000). Physicians and the pharmaceutical industry. Is a gift ever just a gift? JAMA, 283(3), 373-380.

Willison, D., \& Wiktorowicz, M. (2001). International experience with pharmaceutical policy: Common challenges and lessons for Canada. CHEPA working paper series. Center for Health Economics and Policy Analysis.

Wright, D. (2004). The drug bargaining game: pharmaceutical regulation in Australia. Journal of Health Economics, 23(4), 785-813.

Zaric, G. S., \& Xie, B. (2009). The impact of two pharmaceutical risk sharing agreements on pricing, promotion, and net health benefits. Value in Health, 12(5), 838-845. http://dx.doi.org/10.1111/j.1524-4733.2009.00510.x

Zhang, H., \& Zaric, G. S. (2011). Promotion and Leakage under a Pharmaceutical Price-Volume Agreement. INFOR: Information Systems and Operational Research, 49(4), 247-253.

Zhang, H., \& Zaric, G. S. (2015). Using price-volume agreements to manage pharmaceutical leakage and off-label promotion. European Journal of Health Economics, 16(7), 747-761. http://dx.doi.org/10.1007/s10198-014-0626-0

Zhang, H., Zaric, G. S., \& Huang, T. (2011). Optimal design of a pharmaceutical price-volume agreement under asymmetric information about expected market size. Production and Operations Management, 20(3), 334-346. http://dx.doi.org/10.1111/J.1937-5956.2011.01219.X 


\section{Appendix}

Proof for Proposition 1

By deriving both sides of equation (1) with respect to $b$, we have

$$
\frac{\partial m^{E, *}}{\partial b}=-\frac{\frac{2 p^{2}}{(m+b)^{3}}\left[1-\alpha(1-F)-\frac{k}{p}\right]+\frac{p^{3} \alpha f}{(m+b)^{4}}}{\frac{2 p^{2}}{(m+b)^{3}}\left[1-\alpha(1-F)-\frac{k}{p}\right]+\frac{p^{3} \alpha f}{(m+b)^{4}}+\frac{a}{Q}} \text {, and }-1<\frac{\partial m}{\partial b}<0 .
$$

By deriving $E[\pi]$ with respect to $b$, we have

$$
\begin{aligned}
& \frac{\partial E\left[\pi^{E, *}\right]}{\partial b}=\frac{Q p^{2}}{(m+b)^{2}}\left[1-\alpha(1-F)-\frac{k}{p}\right]>0 . \\
& \frac{\partial m^{E, *}}{\partial p}=\frac{\frac{2 p}{(m+b)^{2}}[1-\alpha(1-F)]+\frac{p^{2} \alpha f}{(m+b)^{3}}-\frac{k}{(m+b)^{2}}}{\frac{2 p^{2}}{(m+b)^{3}}\left[1-\alpha(1-F)-\frac{k}{p}\right]+\frac{p^{3} \alpha f}{(m+b)^{4}}+\frac{a}{Q}}>0,
\end{aligned}
$$

and $\frac{\partial E\left[\pi^{E, *}\right]}{\partial p}=E[N]-\alpha E\left[(N-T)^{+}\right]-\frac{Q p}{m+b}\left[1-\alpha(1-F)-\frac{k}{p}\right]$, which may be positive or negative.

$$
\frac{\partial m^{E,{ }^{*}}}{\partial a}=-\frac{m}{\frac{2 Q p^{2}}{(m+b)^{3}}\left[1-\alpha(1-F)-\frac{k}{p}\right]+\frac{Q p^{3} \alpha f}{(m+b)^{4}}+a}<0 \text { and } \frac{\partial E\left[\pi^{*}\right]}{\partial a}=-\frac{m^{2}}{2}<0
$$$$
\frac{\partial m^{E, *}}{\partial \theta_{0}}=-\frac{\frac{\alpha p^{2} f}{(m+b)^{2}}}{\frac{2 p^{2}}{(m+b)^{3}}\left[1-\alpha(1-F)-\frac{k}{p}\right]+\frac{p^{3} \alpha f}{(m+b)^{4}}+\frac{a}{Q}}<0 \text { and } \frac{\partial E\left[\pi^{E, *}\right]}{\partial \theta_{0}}=Q p\left[1-\alpha(1-F)-\frac{k}{p}\right]>0 .
$$$$
\frac{\partial m^{E, *}}{\partial T}=\frac{\frac{\alpha p^{2} f}{(m+b)^{2}}}{\frac{2 p^{2}}{(m+b)^{3}}\left[1-\alpha(1-F)-\frac{k}{p}\right]+\frac{p^{3} \alpha f}{(m+b)^{4}}+\frac{a}{Q}}>0 \text { and } \frac{\partial E\left[\pi^{E, *}\right]}{\partial T}=Q \alpha p(1-F)>0 .
$$

$$
\frac{\partial m^{E,{ }^{*}}}{\partial k}=-\frac{\frac{p}{(m+b)^{2}}}{\frac{2 p^{2}}{(m+b)^{3}}\left[1-\alpha(1-F)-\frac{k}{p}\right]+\frac{p^{3} \alpha f}{(m+b)^{4}}+\frac{a}{Q}}<0 \quad \text { and } \quad \frac{\partial E\left[\pi^{E, *}\right]}{\partial k}=-E[N]<0
$$




$$
\frac{\partial m^{E, *}}{\partial \alpha}=-\frac{\frac{p^{2}(1-F)}{(m+b)^{2}}}{\frac{2 p^{2}}{(m+b)^{3}}\left[1-\alpha(1-F)-\frac{k}{p}\right]+\frac{p^{3} \alpha f}{(m+b)^{4}}+\frac{a}{Q}}<0 \quad \text { and } \quad \frac{\partial E\left[\pi^{E, *}\right]}{\partial \alpha}<0 .
$$

\section{Proof for Proposition 2}

$$
\begin{aligned}
& \frac{\partial^{2} \mathrm{E}[\pi]}{\partial m^{2}}=-\frac{2 Q p^{2}}{(m+b)^{3}}[1-\alpha(1-F)]-\frac{Q^{2} \alpha p^{3}}{(m+b)^{4}} f-a, \\
& \frac{\partial^{2} \mathrm{E}[\pi]}{\partial p^{2}}=-\frac{2 Q}{m+b}[1-\alpha(1-F)]-\frac{Q^{2} \alpha p}{(m+b)^{2}} f, \text { and } \\
& \frac{\partial^{2} \mathrm{E}[\pi]}{\partial m \partial p}=\frac{2 Q p}{(m+b)^{2}}[1-\alpha(1-F)]+\frac{Q^{2} \alpha p^{2} f}{(m+b)^{3}} .
\end{aligned}
$$

Thus, the Hessian determinant is $A=\frac{\partial^{2} \mathrm{E}[\pi]}{\partial m^{2}} \frac{\partial^{2} \mathrm{E}[\pi]}{\partial p^{2}}-\left(\frac{\partial^{2} \mathrm{E}[\pi]}{\partial m \partial p}\right)^{2}>0$.

$\frac{\partial^{2} \mathrm{E}[\pi]}{\partial m \partial b}=-\frac{2 Q p^{2}}{(m+b)^{3}}[1-\alpha(1-F)]-\frac{Q^{2} \alpha p^{3} f}{(m+b)^{4}}$ and $\frac{\partial^{2} \mathrm{E}[\pi]}{\partial p \partial b}=\frac{2 Q p}{(m+b)^{2}}[1-\alpha(1-F)]+\frac{Q^{2} \alpha p^{2} f}{(m+b)^{3}}$.

Thus, $\quad \frac{d m}{d b}=\frac{-\frac{\partial^{2} \mathrm{E}[\pi]}{\partial p^{2}} \frac{\partial^{2} \mathrm{E}[\pi]}{\partial m \partial b}+\frac{\partial^{2} \mathrm{E}[\pi]}{\partial m \partial p} \frac{\partial^{2} \mathrm{E}[\pi]}{\partial p \partial b}}{A}=0, \quad$ and $\quad \frac{d p}{d b}=\frac{-\frac{\partial^{2} \mathrm{E}[\pi]}{\partial m^{2}} \frac{\partial^{2} \mathrm{E}[\pi]}{\partial p \partial b}+\frac{\partial^{2} \mathrm{E}[\pi]}{\partial p \partial m} \frac{\partial^{2} \mathrm{E}[\pi]}{\partial m \partial b}}{A}=$

$$
\frac{\frac{2 Q a p}{(m+b)^{2}}[1-\alpha(1-F)]+\frac{Q^{2} a \alpha p^{2} f}{(m+b)^{3}}}{A}>0
$$

$$
\frac{\partial^{2} \mathrm{E}[\pi]}{\partial m \partial T}=\frac{Q p^{2} \alpha f}{(m+b)^{2}} \text { and } \frac{\partial^{2} \mathrm{E}[\pi]}{\partial p \partial T}=\alpha(1-F)-\frac{Q \alpha p f}{m+b} .
$$$$
-\frac{\partial^{2} \mathrm{E}[\pi]}{\partial p^{2}} \frac{\partial^{2} \mathrm{E}[\pi]}{\partial m \partial T}+\frac{\partial^{2} \mathrm{E}[\pi]}{\partial m \partial p} \frac{\partial^{2} \mathrm{E}[\pi]}{\partial p \partial T}=\frac{Q \alpha p(1-F)}{(m+b)^{2}}\left\{2[1-\alpha(1-F)]+\frac{Q \alpha p f}{m+b}\right\}>0 .
$$$$
-\frac{\partial^{2} \mathrm{E}[\pi]}{\partial m^{2}} \frac{\partial^{2} \mathrm{E}[\pi]}{\partial p \partial T}+\frac{\partial^{2} \mathrm{E}[\pi]}{\partial p \partial m} \frac{\partial^{2} \mathrm{E}[\pi]}{\partial m \partial T}=Q \alpha(1-F)\left\{\frac{2 p^{2}}{(m+b)^{3}}[1-\alpha(1-F)]+\frac{\alpha p^{3}}{(m+b)^{4}} f+a\right\}-\frac{Q a \alpha p f}{m+b} \quad, \quad \text { which }
$$

may be positive or negative.

$$
\frac{\partial^{2} \mathrm{E}[\pi]}{\partial m \partial \theta_{0}}=-\frac{Q^{2} p^{2} \alpha f}{(m+b)^{2}} \text { and } \frac{\partial^{2} \mathrm{E}[\pi]}{\partial p \partial \theta_{0}}=\frac{Q^{2} p \alpha f}{m+b}+Q[1-\alpha(1-F)] .
$$


Thus, $\quad-\frac{\partial^{2} \mathrm{E}[\pi]}{\partial p^{2}} \frac{\partial^{2} \mathrm{E}[\pi]}{\partial m \partial \theta_{0}}+\frac{\partial^{2} \mathrm{E}[\pi]}{\partial m \partial p} \frac{\partial^{2} \mathrm{E}[\pi]}{\partial p \partial \theta_{0}}=\frac{Q p[1-\alpha(1-F)]}{(m+b)^{2}}\left\{2[1-\alpha(1-F)]+\frac{\alpha p f}{m+b}\right\} \quad>0, \quad$ and $-\frac{\partial^{2} \mathrm{E}[\pi]}{\partial m^{2}} \frac{\partial^{2} \mathrm{E}[\pi]}{\partial p \partial \theta_{0}}+\frac{\partial^{2} \mathrm{E}[\pi]}{\partial p \partial m} \frac{\partial^{2} \mathrm{E}[\pi]}{\partial m \partial \theta_{0}}=[1-\alpha(1-F)]\left\{\frac{2 p^{2}}{(m+b)^{3}}[1-\alpha(1-F)]+\frac{\alpha p^{3}}{(m+b)^{4}} f+a\right\}+\frac{a \alpha p f}{m+b}>0$. Thus, $\frac{d m}{d \theta_{0}}>0$ and $\frac{d p}{d \theta_{0}}>0$

$\frac{\partial^{2} \mathrm{E}[\pi]}{\partial m \partial a}=-m$ and $\frac{\partial^{2} \mathrm{E}[\pi]}{\partial p \partial a}=0$. Thus, $\frac{d m}{d a}<0$, and $\frac{d p}{d a}<0$.

$\frac{\partial^{2} \mathrm{E}[\pi]}{\partial m \partial \alpha}=-\frac{Q^{2} p^{2}(1-F)}{(m+b)^{2}}$ and $\frac{\partial^{2} \mathrm{E}[\pi]}{\partial p \partial \alpha}=-E\left[(N-T)^{+}\right]+\frac{Q p(1-F)}{m+b}$.

Thus, $-\frac{\partial^{2} \mathrm{E}[\pi]}{\partial p^{2}} \frac{\partial^{2} \mathrm{E}[\pi]}{\partial m \partial \alpha}+\frac{\partial^{2} \mathrm{E}[\pi]}{\partial m \partial p} \frac{\partial^{2} \mathrm{E}[\pi]}{\partial p \partial \alpha}=-E\left[(N-T)^{+}\right]\left\{\frac{2 Q p}{(m+b)^{2}}[1-\alpha(1-F)]+\frac{Q^{2} \alpha p^{2} f}{(m+b)^{3}}\right\}$ and $-\frac{\partial^{2} \mathrm{E}[\pi]}{\partial m^{2}} \frac{\partial^{2} \mathrm{E}[\pi]}{\partial p \partial \alpha}+\frac{\partial^{2} \mathrm{E}[\pi]}{\partial p \partial m} \frac{\partial^{2} \mathrm{E}[\pi]}{\partial m \partial \alpha}=\frac{\partial^{2} \mathrm{E}[\pi]}{\partial m^{2}} E\left[(N-T)^{+}\right]+\frac{a Q p(1-F)}{m+b} . \frac{d m}{d \alpha}<0$ and $\frac{d p}{d \alpha}$ can be positive or negative. 